\title{
Comparative Study between Plain Radiography and Ultrasound Abdomen in Non Traumatic Surgical Acute Abdominal Conditions
}

\author{
Sharma $\mathbf{P}^{1}$, Sidharth ${ }^{2}$, Singh $B P^{3}$, Singh $D^{3}$, Gupta $A^{4}$ \\ ${ }^{1}$ Department of Radiology and Imaging, Manipal Teaching Hospital, Pokhara, Nepal, \\ ${ }^{2}$ Department of General Surgery, ${ }^{3}$ Department of Radiology and Imaging, Nepalgunj Medical \\ College Teaching Hospital, Kohalpur, Nepal, ${ }^{4}$ Department of Radio diagnosis \& Imaging, \\ B.P. Koirala Institute of Health Sciences, Dharan, Nepal
}

\begin{abstract}
Introduction: Acute abdomen might indicate a progressive intra-abdominal condition that is threatening to life or capable of causing severe morbidity. A good history, thorough clinical examination, laboratory investigations and imaging studies is necessary in order to arrive at a correct diagnosis. The aim of our study was to compare the diagnostic yield of traditional three-view abdominal x-ray series (upright chest $\mathrm{x}$-ray, supine and upright abdominal x-rays) with that of ultrasound in patients presenting with non traumatic acute abdominal surgical pain. Methods: This prospective study was conducted between February and July 2010 on 65 consecutive patients. Critically ill patients, pregnant ladies, patient with trauma abdomen, acute abdomen due to gynaecological pathologies were excluded from our study. Detail abdominal ultrasound was done. After ultrasound traditional three-views acute abdominal xray series (AAS) were taken. Final diagnosis was made on the basis of operative findings/therapeutic response/ histopathological/ laboratory findings. Finally, accuracy of clinical diagnosis, plain film and ultrasound in evaluation of acute abdomen was determined. Results: Ultrasound yielded an overall sensitivity and specificity of $78.7 \%$ and $84.6 .6 \%$ respectively. The AAS interpretations yielded an overall sensitivity and specificity of $23.4 \%$ and $38.40 \%$ respectively. Conclusion: Plain $\mathrm{x}$ ray is less sensitive in the evaluation of nontraumatic acute abdomen so it should be used together with ultrasound abdomen in order to arrive at a correct diagnosis.
\end{abstract}

Keywords: Acute abdomen, Ultrasonography, X-ray abdomen

\section{Introduction}

The term 'acute abdomen' is a loose one encompassing all those conditions that present with clinical features of short duration (arbitrarily less than 10 days) which might indicate a progressive intra-abdominal condition that is threatening to life or capable

Correspondence to : Dr. Prakash Sharma,MD Department of Radiology and Imaging, Manipal Teaching Hospital, Pokhara, Nepal E mail: prakashshrm@yahoo.com 
of causing severe morbidity. ${ }^{1}$ The majority of such patients have pain as their chief symptoms. Acute abdomen does not invariably signify the need for surgical intervention.

A good history, thorough clinical examination, laboratory investigations and imaging studies is necessary in order to arrive at a correct diagnosis so that timely management can be done.

Studies have shown that abdominal radiographs are not sensitive in the evaluation of adult patients presenting with nontraumatic abdominal pain. ${ }^{2,3}$ Ultrasound is easily available and lacks radiation. The purpose of this study was to prospectively evaluate the diagnostic accuracy of Ultrasound in patients with nontraumatic acute abdominal pain as compared with traditional abdominal radiography.

\section{Methods}

This prospective study was conducted between February and July 2010 on 65 consecutive patients referred to the department of radiology and Imaging from the department of emergency, Nepalgunj Medical College Teaching Hospital Kohalpur for evaluation of acute abdomen. Critically ill patients, pregnant ladies, patient with trauma abdomen, acute abdomen due to gynaecological pathologies were excluded from our study. After obtaining consent clinical information were recorded in preformed Performa.

Detail abdominal ultrasound was done. After ultrasound traditional three-views (upright chest $\mathrm{x}$-ray, supine and upright abdominal $\mathrm{x}$ rays) acute abdominal $\mathrm{x}$-ray series (AAS) were taken. Left lateral decubitus film was taken only when required. The patient was kept in a given position for 10 minutes before the horizontal-ray radiograph to allow time for any free gas to rise to the highest point. The bladder was emptied before the supine radiograph was taken and the area from the diaphragm to the hernial orifices was included in the film.

Plain X-rays were evaluated by a blinded (DS) radiologist. The images were interpreted with only the knowledge that patients presented with abdominal pain. Ultrasound was done by a blinded radiologist (PS). If free fluid or collection was seen in the peritoneal cavity ultrasound guided aspiration was done.

Special investigation like intravenous urography, contrast studies of gastrointestinal tract, CT scan of abdomen were conducted whenever necessary.

Final diagnosis was made on the basis of operative findings/therapeutic response/ histopathological/laboratory findings. These data were analysed manually to meet the objectives of the study.

\section{Results}

This prospective study was conducted in 65 acute abdomen patients referred to department of radiology for $\mathrm{x}$-ray and ultrasound from emergency. Out of 65 patients, five were excluded due to gynaecological pathology, four were excluded due to pregnancy and two patients who were critically ill were excluded from our study. Remaining 54 patients were included for statistical analysis. Age of the patient ranged from 5 months to 75 years. Most of the patients in our study were in the age group 31-40 years. Mean age of the patients was 37.9 yrs \pm 16.7 years. Most of 
the patients in our study were male $(59.3 \%)$. Male: Female ratio was 1.45:1. The most common chief complaint was acute abdominal pain in 53 cases $(98.1 \%)$. Other presenting symptom included vomiting $(0.01 \%)$.

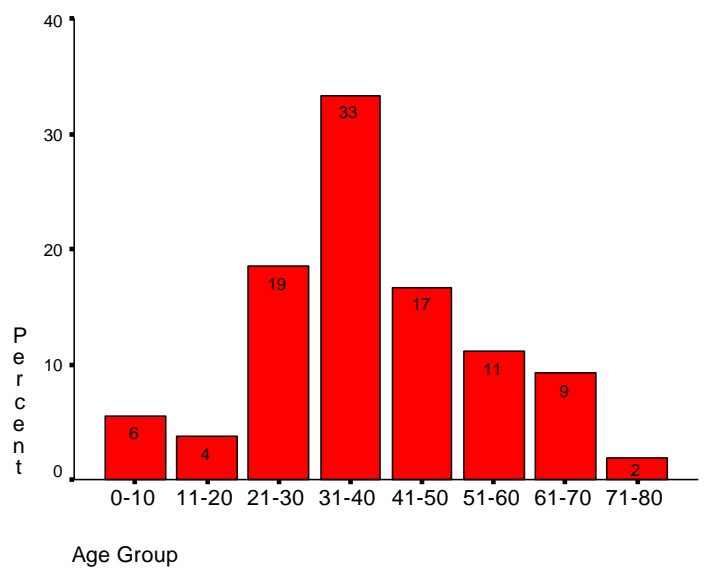

Fig 1: Age distribution of the patients.

Based on the clinical examination, $12(22.2 \%)$ cases were diagnosed as acute appendicitis, $10(18.5 \%)$ as urolithiasis, $8(14.8 \%)$ as acute cholecystitis, $5(9.2 \%)$ as acute pancreatitis, $4(7.4 \%)$ as acute intestinal obstruction, $3(5.5 \%)$ as liver abscess and $3(5.5 \%)$ as peritonitis due to bowel perforation. There were 2 cases $(3.7 \%)$ each of peptic ulcer, pelvic abscess and psoas abscess. There were one case each of hypertrophic pyloric stenosis, colitis and acute pyelonephritis.

Based on the ultrasound diagnosis, $9(16.6 \%)$ were diagnosed as urolithiasis, $8(14.8 \%)$ as acute cholecystitis, $7(12.9 \%)$ as acute appendicitis, $5(9.2 \%)$ as bowel perforation, $4(7.4 \%)$ as acute pancreatitis, $4(7.4 \%)$ as liver abscess, $2(3.7 \%)$ as psoas abscess, $2(3.7 \%)$ as pelvic abscess and one case each of superior mesenteric vein thrombosis, mesenteric lymphadenopathy, acute pyelonephritis, intestinal obstruction., intestinal ascariasis and biliary peritonitis due to gall bladder perforation. However, seven cases on ultrasound examination had a normal scan.

Based on the evaluation of plain x ray, bowel perforation was diagnosed in $5(9.2 \%)$ cases, acute pancreatitis in $1(1.8 \%)$, intestinal obstruction in $3(5.5 \%)$, urolithiasis in 8 (14.8\%) and potts spine with psoas abscess in $1(1.8 \%)$ case. Radiographs of $36(66.66 \%)$ patients were normal or with nonspecific findings.

On the basis of operative findings/ therapeutic response/ histopathological/ laboratory findings final diagnosis was made. Most of the cases, that is, $19(35.18 \%)$ were of gut pathology (Table 1).

Table 1: Distribution of cases based on final diagnosis.

\begin{tabular}{|l|l|}
\hline Diagnosis & Percentage \\
\hline Gut Pathology & $\mathbf{1 9 ( 3 5 . 1 8 \% )}$ \\
\hline Hepatobiliary Pathology & $\mathbf{1 2 ( 2 2 . 2 2 \% )}$ \\
\hline Pancreatic Pathology & $\mathbf{4 ( 7 . 4 \% )}$ \\
\hline Renal & $\mathbf{1 1 ( 2 0 . 3 \% )}$ \\
\hline Others & $\mathbf{8 ( 1 4 . 4 \% )}$ \\
\hline \hline Total & $\mathbf{5 4}$ \\
\hline
\end{tabular}

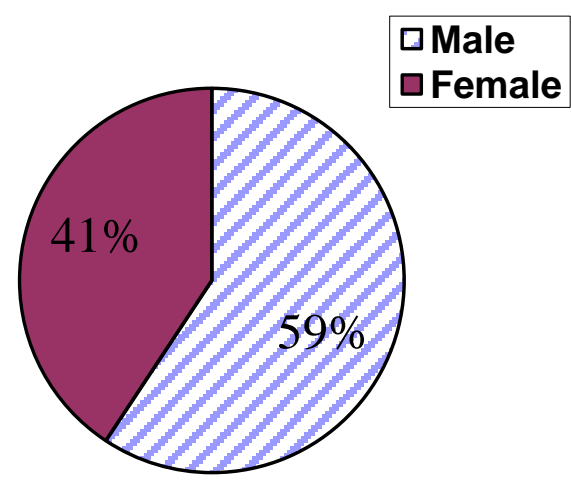

Fig 2: Sex wise distribution of the patients. 
The sensitivity and specificity of ultrasound in the diagnosis of acute appendicitis, acute cholecystitis, GIT perforation, Liver abscess, Pelvic abscess and psoas abscess was $100 \%$. Similarly, Plain $\mathrm{X}$ ray had sensitivity and specificity of $100 \%$ in the diagnosis of GIT perforation and intestinal obstruction. The overall sensitivity and specificity of ultrasound was higher than plain $\mathrm{x}$ ray in the diagnosis of acute abdomen (Table 2).

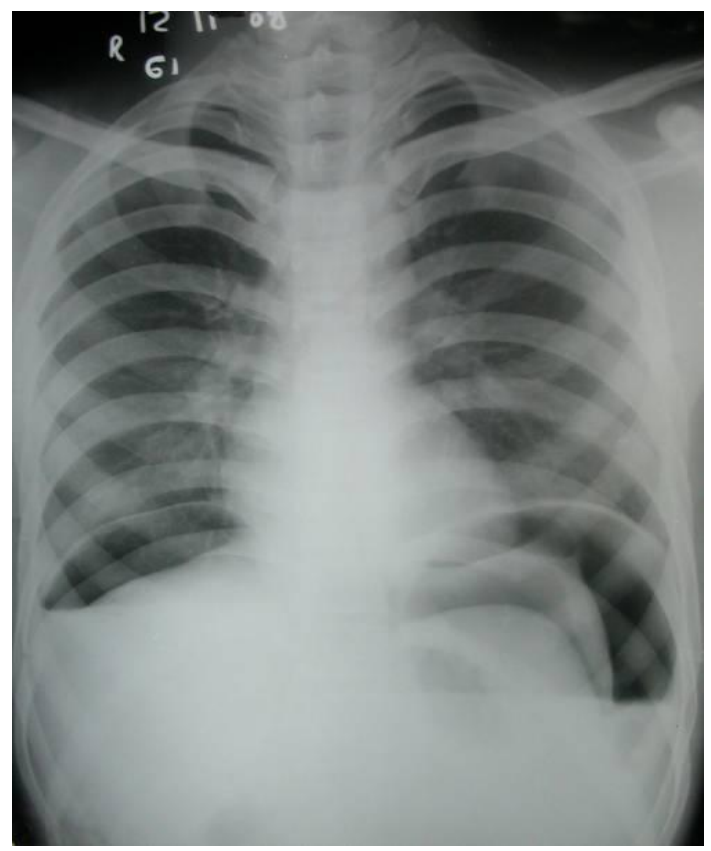

Fig 3: Chest $x$ ray PA view showing air under the diaphragm suggestive of pneumoperitoneum.

Table 2: Overall sensitivity and specificity of ultrasound and plain $x$ ray in the diagnosis of acute abdomen.

\begin{tabular}{|lll|} 
& Sensitivity & Specificity \\
\hline Ultrasound & $78.7 \%$ & $84.6 .6 \%$ \\
\hline Plain X ray & $23.4 \%$ & $38.40 \%$ \\
\hline
\end{tabular}

\section{Discussion}

The accurate diagnosis of acute abdominal conditions is crucial in the management of patients in emergency. The varieties of conditions requiring immediate management vary widely in their clinical presentation and laboratory findings. Various pathological processes presenting with acute abdomen can be grouped into four groups. ${ }^{4}$

Right upper quadrant:

a) Acute cholecystitis

b) Acute pancreatitis

c) Renal and ureteric colic

d) Acute retrocecal appendicitis

e) Supradiaphragmatic causes

- Consolidation

- Pleural effusion

Right lower quadrant:

a) Acute appendicitis

b) Renal and ureteric colic

c) Ectopic pregnancy

d) Ovarian torsion

e) Hemorrhagic ovarian cyst

f) Diverticulitis

g) Pelvic inflammatory disease

h) Psoas abscess

i) Intussusception

Left upper quadrant:

a) Acute pancreatitis

b) Subphrenic abscess

c) Splenic abscess

d) Renal and ureteric colic

e) Left pleural effusion/consolidation

f) Myocardial infarction

g) Ruptured aneurysm

Left lower quadrant:

a) Renal and ureteric colic 

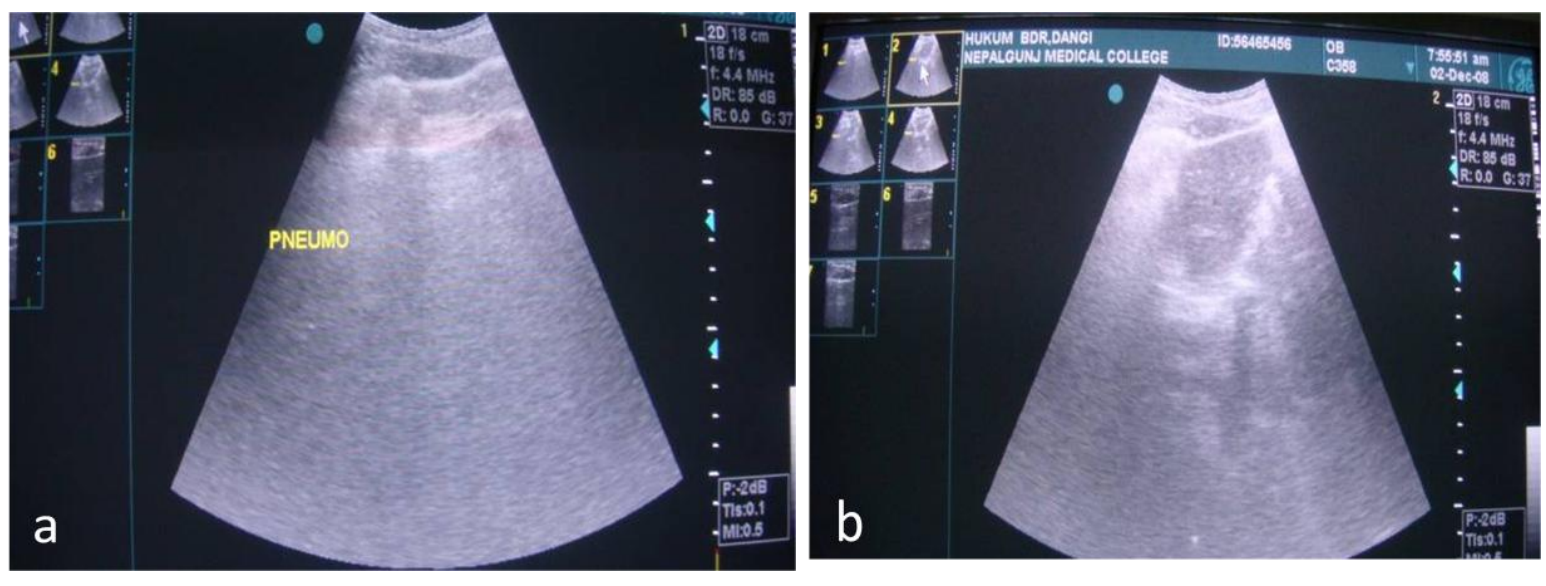

Figure 4 a \&b: Ultrasound of upper abdomen showing enhancement of peritoneal stripe with distal reverberations suggestive of pneumoperitoneum.
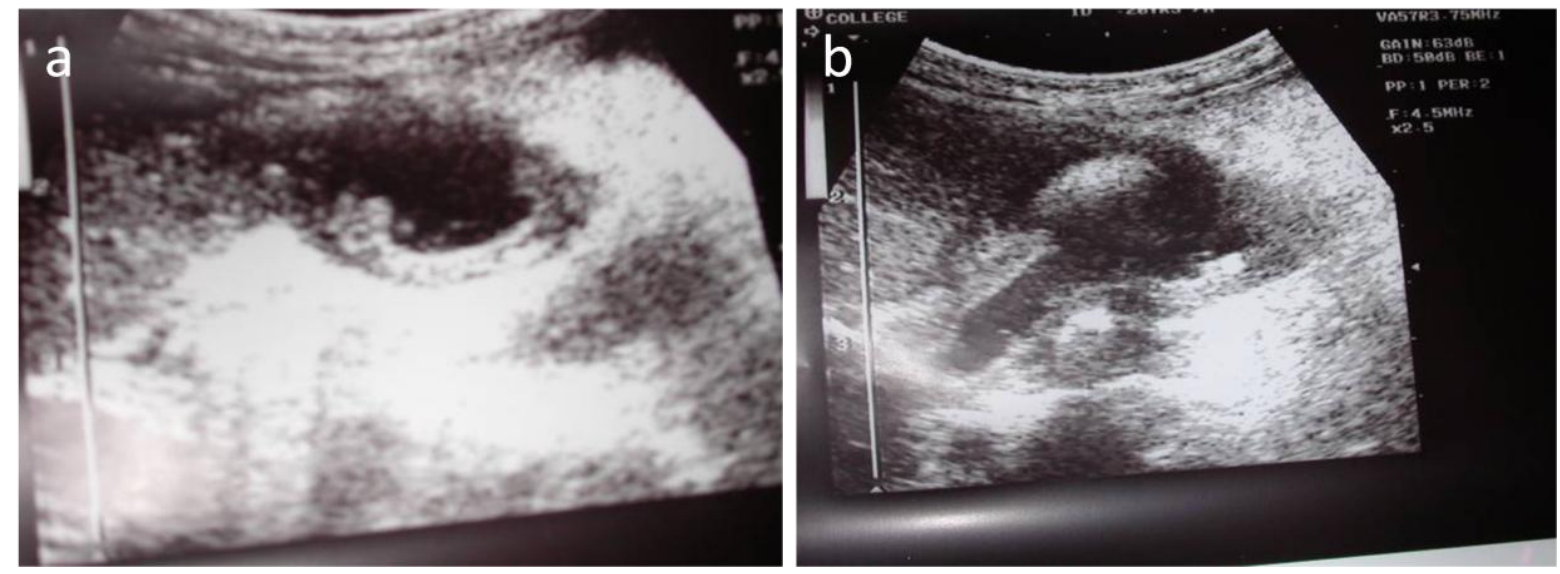

Fig 5 a \& b: Ultrasound of the gall bladder showing a small defect in the wall of gall bladder with a calculus lying outside the gall bladder between gall bladder and liver.

b) Ectopic pregnancy

c) Ovarian torsion

d) Hemorrhagic ovarian cyst

e) Pelvic inflammatory disease

f) Diverticulitis

g) Ruptured aneurysm

h) Intussusception

Abdominal radiography has historically been the first imaging examination performed in the emergency department in evaluating abdominal pain. Interpretation of these radiographs may present a formidable challenge to the radiologist. While in many cases a specific diagnosis can be made, plain radiographs are often nonspecific or even misleading. Other imaging modalities like Ultrasound and computed tomography (CT) are used increasingly in cases where there is diagnostic difficulty or clinical uncertainty. Present study was aimed to compare the sensitivity and specificity of ultrasound and plain film in the diagnosis of non traumatic acute abdominal conditions.

In our series, $19(35.18 \%)$ cases were of gut pathology. This was followed by hepatobiliary pathology, 12(22.22\%) cases. In contrast to this, Gupta $\mathrm{K}$ et al and Walsh 
PF et al had found that most of the cases in their series were of hepatobiliary origin. ${ }^{5,6}$

The overall sensitivity and specificity of ultrasound was $78.7 \%$ and $84.66 \%$ respectively, which is slightly lower than Gupta K et al. ${ }^{5}$ Similarly overall sensitivity and specificity of plain $\mathrm{x}$ ray was $23.4 \%$ and $38.4 \%$ respectively which is also lower than that of Gupta K et al. ${ }^{5}$

In a retrospective study of 1000 patients with nontraumatic acute abdominal pain, Ahn et al concluded "abdominal radiographs are not sensitive in the evaluation of adult patients presenting to the emergency department with nontraumatic abdominal pain. ${ }^{2}$ Other series have also concluded that abdominal radiography is of limited use in the assessment of patients with acute abdominal pain which is similar to our series. ${ }^{7,8}$

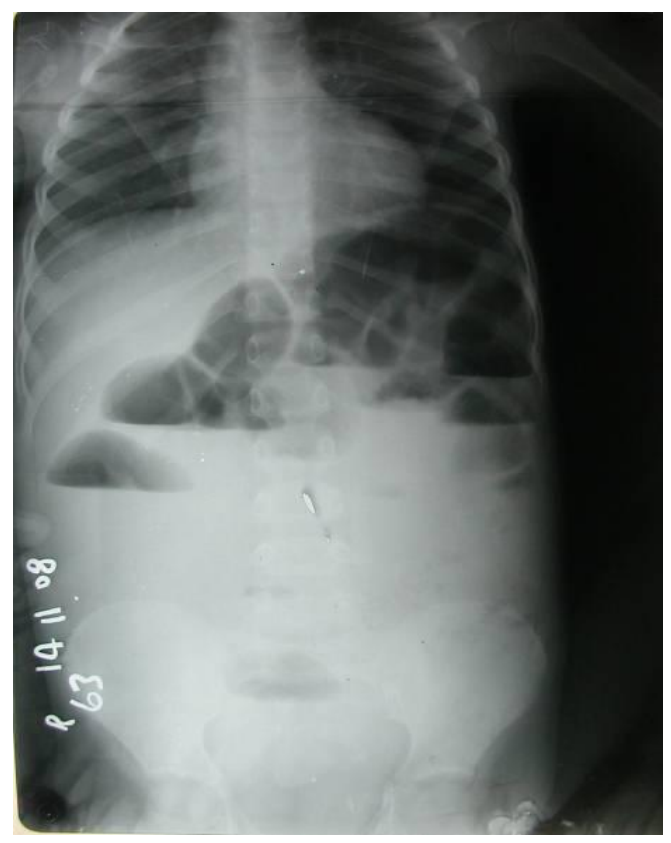

Fig 6: Erect X-ray abdomen showing multiple air fluid level suggestive of small bowel obstruction.

Ultrasonography plays an important role in evaluating patients of suspected acute cholecystitis with a reported accuracy of 95$99 \%$.

On the basis of ultrasound, most authorities diagnose acute cholecystitis if there are gallstones and the gallbladder if focally tender. A sonographic Murphy's sign is present when maximal tenderness is elicited over the sonographically localized gallbladder. Impacted gallstone is another primary sign. Other secondary sonographic criteria that are sensitive but less specific for diagnosing acute cholecystitis include gallbladder dilatation, sludge and diffuse wall thickening. ${ }^{10}$

A plain radiography shows an incidence of 10 to $15 \%$ radiopaque gall stones. ${ }^{11}$ In the present study none of our patients had radiopaque gall stone. Ultrasound (USG) is as effective as radiography in the diagnosis of pneumoperitoneum. ${ }^{12-15}$ The main sonographic signs of the presence of pneumoperitoneum include strong distal reverberations, the shifting phenomenon, and the enhancement of peritoneal stripes. The scissors maneuver may be a useful adjunct for improving the diagnostic yield of sonography. ${ }^{16}$

Ultrasonography has reported sensitivities of 75 to $90 \%$ and specificities of 86 to 100 percentages in diagnosing acute appendicitis. ${ }^{17}$ In our study it was around $100 \%$. This was due to the small number of cases with acute appendicits. Puylaert's technique of using graded compression is the most popular method of examination. ${ }^{18}$ The inflammed appendix is seen as a blind ending laminated, non compressible, tubular structure $>6 \mathrm{~mm}$ in diameter arising from the base of the caecum. Appendicoliths are seen as bright echogenic foci with clean distal acoustic shadowing. ${ }^{17}$ 
There was one case of infantile hypertrophic pyloric stenosis which was diagnosed by ultrasound and confirmed by surgery. Plain X-ray abdomen was not helpful in diagnosing this condition. Sonographically, thickness of hypertrophied muscle from serorsa to mucosa is considered the most reliable measurement for the diagnosis of infantile hypertrophic pyloric stenosis. Muscle thickness of $4 \mathrm{~mm}$ or more, diameter of hypertrophied segment $>15 \mathrm{~mm}$ and length of hypertrophied segment of pylorus $>17 \mathrm{~mm}$ was considered diagnostic of hypertrophic pyloric stenosis. On transverse section, the pylorus has an appearance of "Target or bull's eye. ${ }^{19}$

One case of superior mesenteric vein thrombosis was diagnosed by the use of colour doppler ultrasound and plain $\mathrm{x}$ ray abdomen was not helpful in the case.

In our study sensitivity and specificity of AAS in diagnosing pneumoperitoneum and intestinal obstruction was $100 \%$ which is similar to Gupta K et al. ${ }^{5}$

Limitations of this study include relatively small sample size and possible selection bias, as the patients were referred by the emergency medical officers at a rate of two or three patients per day.

\section{Conclusion}

In conclusion, AAS is a less sensitive technique in the evaluation of nontraumatic acute abdomen. It should be used together with ultrasound abdomen in order to arrive at a correct diagnosis

\section{References}

1. Spigelman AD. Acute abdominal conditions. In: Henry MM, Thompson JN editors. Clinical surgery $2^{\text {nd }}$ edition. China: Elsevier Saunders.2005.365-366.

2. Joshi MS. Ultrasonography of the acute abdomen [online]. 1997 [cited 2009 May 5]; Available from: url:http:// www. Starprogram.de/data--star-program/upload/ star_abstracts_180_joshi-acute-abdomen .pdf

3. Ahn SH, Mayo-Smith WW, Murphy BL, Reinert SE, Cronan JJ. Acute nontraumatic abdominal pain in adult patients: abdominal radiograhy compared with CT evaluation. Radiology 2002; 225:159-164.

4. MacKersie AB, Lane MJ, Gerhardt RT, Claypool HA, Keenan S, Katz DS et al. Nontraumatic acute abdominal pain: Unenhanced helical CT compared with three-view acute abdominal series. Radiology 2005 Oct; 237:114-22.

5. Gupta K, Bhandari RK, Chander R. Comparative study of plain abdomen and ultrasound in non-traumatic acute abdomen. Indian J Radiol Imaging 2005; 15:109-15.

6. Walsh PF, Crossling F. The value of immediate ultrasound in acute abdomen conditions: A critical appraisal. Clin Radiol 1990; 42: 47-49.

7. Ralls PW, Colletti PM, Lapin SA. Real time sonography in suspected acute cholecystitis. Radiology 1985; 155: 767 771.

8. Laing FC. The gall bladder and bile duct. In: Carol MR, Stephanie RW, William C, 
editors. Diagnostic ultrasound. 2nd ed. St. Louis: Mosby; 1998. 186-190.

9. Rosenquist CJ. Radiology of biliary tree. Surg Clin North Amer 1981; 61 (4):775862.

10. Chang-Chien CS, Lin HH, Yen CL. Sonographic demonstration of free air in perforated peptic ulcers: comparison of sonography with radiography. J Clin Ultrasound 1989; 17:95.

11. Nirapathpongporn S, Osatavanichvong K, Udompanich O. Pneumoperitoneum detected by ultrasound. Radiology 1984; 150:831.

12. Lee DH, Lim JH, Ko YT. Sonographic detection of pneumoperitoneum in patients with acute abdomen. AJR Am J Roentgenol 1990; 154:107.

13. Muradali D, Wilson S, Burns PN. A specific sign of pneumoperitoneum on sonography: enhancement of the peritoneal stripe. AJR Am J Roentgenol 1999; 173:1257.

14. Karahan O I, Kurt A, Yikilmaz A, Kahriman G. New Method for the
Detection of Intraperitoneal Free Air by Sonography:Scissors Maneuver. J Clin Ultrasound 2004; 32:381-385.

15. Garg A. Imaging of Appendix. In: Berry M,Chaudhary V,Mukhopadhyay S,Suri S editors. Diagnostic Radiology Gastrointestinal and hepatobiliary imaging. 2nd ed. New Delhi: Jaypee; 2004.200-214.

16. Puylaert JB. Acute appendicitis. US evaluation using graded compression. Radiology 1986; 158:3551-35560.

17. Hudson AP, Susan BP. Emergency Med. Clinics of North Amer 1997; 15 (4): 825846.

18. Eisenberg RL, Heinken P, Hedgcock MW, Federle M, Goldberg HI. Evaluation of plain abdominal radiographs in the diagnosis of abdominal pain. Ann Surg 1983; 197:464-469.

19. Campbell JP, Gunn AA. Plain abdominal radiographs and acute abdominal pain. Br J Surg 1988; 75:554-556. 